\title{
Absence of phase-dependent noise in time-domain reflectivity studies of impulsively excited phonons
}

\author{
A. Hussain* and S. R. Andrews ${ }^{\dagger}$ \\ Department of Physics, University of Bath, Bath BA2 7AY, United Kingdom \\ (Received 1 April 2010; revised manuscript received 24 May 2010; published 17 June 2010)
}

\begin{abstract}
There have been several reports of phase-dependent noise in time-domain reflectivity studies of optical phonons excited by femtosecond laser pulses in semiconductors, semimetals, and superconductors. It was suggested that such behavior is associated with the creation of squeezed phonon states although there is no theoretical model that directly supports such a proposal. We have experimentally re-examined the studies of phonons in bismuth and gallium arsenide, and find no evidence of any phase-dependent noise signature associated with the phonons. We place an upper limit on any such noise at least 40-50 dB lower than previously reported.
\end{abstract}

DOI: 10.1103/PhysRevB.81.224304

PACS number(s): 78.47.jg, 63.20.-e, 42.50.Lc

Coherent phonons in semiconductors, superconductors, metals, and insulators have been widely studied in the time domain since the first report of their excitation by femtosecond laser pulses in about 1990.1,2 The most general excitation mechanism is impulsive stimulated Raman scattering (ISRS). ${ }^{3}$ A special case of ISRS in opaque materials is sometimes referred to as the displacive mechanism ${ }^{1}$ and is associated with electronic excitation from bonding to antibonding orbitals. Longitudinal optical phonons in semiconductors can also be driven by the transient polarization associated with the screening of surface space-charge fields following interband excitation. $^{2}$

The amplitude of a phonon mode may be decomposed into two quadrature components with the time dependences $\cos \omega t$ and $\sin \omega t$. In a coherent state, the closest quantum counterpart to a classical field, the fluctuations in the two quadratures have the same variance and are randomly distributed in phase. The product of the variances in each quadrature is the minimum allowed by the Heisenberg uncertainty principle. Minimum uncertainty states in which the fluctuations in one quadrature have a variance that falls below the zero-point quantum noise level are called squeezed states. ${ }^{4}$ Squeezed electromagnetic fields were experimentally studied for the first time in the mid-1980s (Ref. 5) and nonclassical light is now an established area of research. ${ }^{6}$ A phasedependent nonlinear process is necessary to generate a squeezed state. For example, squeezed optical fields can be produced by parametric amplification or four-wave mixing. Recently, there has been interest in extending the study of nonclassical fields to lattice vibrations in condensed matter but except for a few studies of squeezing, ${ }^{7-12}$ and amplitude collapse and revival, ${ }^{13}$ there has been relatively little work on phonon fields. Squeezing of phonons generated by secondorder Raman scattering was predicted ${ }^{7}$ and later reported ${ }^{9,11}$ in low temperature, femtosecond pump-probe transmission measurements on $\mathrm{KTaO}_{3}$. In this case the mechanism is a three-phonon parametric process analogous to that used to produce coherent two-photon states. This type of experiment has been extended to the creation of coexcited coherent and squeezed vibrations ${ }^{10}$ and to squeezed spin waves ${ }^{14}$ in $\mathrm{MnF}_{2}$ but in no case has the noise been unequivocally shown to fall below the quantum limit because of the difficulty of making direct optical measurements of the variance of atomic displacements in condensed matter. ${ }^{8}$ More recently, femtosecond x-ray diffraction has been used to probe the statistics of lattice vibrations following intense (several millijoule per square centimeter) optical excitation of single crystal $\mathrm{Bi}^{12}$ Analysis of the time-dependent Debye-Waller factors provided what appears to be the first direct observation of phonon squeezing. In this case the mechanism is associated with the step change in phonon frequency induced by electronic excitation.

In a very different and more controversial, type of alloptical experiment, Misochko et al. ${ }^{15-18}$ have reported the observation of a phase-dependent variance in the probe intensity in room temperature, time-domain reflectivity studies of phonons impulsively excited by relatively weak (few tens of microjoule per square centimeter) femtosecond laser pulses. These studies encompass a wide range of materials, including the semiconductor GaAs, ${ }^{15}$ semimetals $\mathrm{Sb}^{16}$ and $\mathrm{Bi}^{15}$ and the high-temperature superconductor $\mathrm{YBa}_{2} \mathrm{Cu}_{3} \mathrm{O}_{7-\delta} \cdot{ }^{17,18}$ The variance in the time-domain reflectivity was found to have maxima at nodes in the phonon contribution and minima at antinodes. The detection of impulsively excited phonons in reflection or transmission experiments relies on the modulation of the dielectric function by atomic displacements via the deformation potential interaction and, in the case of polar phonons, also by longitudinal electric fields. The phase-dependent noise was therefore tentatively ascribed to the creation of squeezed rather than coherent phonons on the assumption that the statistics of the probing photon field reflects that of the probed phonon field, although there is no reference signal that allows direct comparison with the quantum noise limit. The results of these experiments are puzzling because it is difficult to understand how phonon excitation mechanisms as different as transient screening and impulsive Raman scattering could lead to similar nonclassical behavior. Indeed, there are no theoretical models that directly account for the creation of a squeezed vibrational state in any of these experiments. The effect is also large and surprisingly similar in a range of different materials with a maximum root-mean-square (rms) noise as a percentage of the peak to phonon oscillation amplitude in the range $15-35 \%{ }^{19}$ Furthermore, squeezed lattice fields are 
generally expected to be continuumlike rather than quasi-monochromatic. ${ }^{10}$ The objective of the present study was to revisit some of the time-resolved reflectivity experiments of Misochko et al., ${ }^{15}$ focusing on those on $\mathrm{Bi}$ and GaAs which typify the reported behavior, in an attempt to clarify the origin of their observations.

In our experiments, phonons were impulsively excited at room temperature in GaAs and $\mathrm{Bi}$ using 810-nm center wavelength pulses from an argon-ion-pumped, 10-fs Ti:sapphire oscillator, and probed by reflective electro-optic sampling (REOS) ${ }^{20}$ The melt-grown Bi sample was polycrystalline and mechanically lapped flat with $1-\mu \mathrm{m}$ diamond paste. The GaAs sample consisted of a $p-i-n$ structure grown by molecular beam epitaxy and described previously. ${ }^{21}$ The large built-in electric field in this sample enhances the phonon signal. In experiments on both samples, a $240 \mathrm{~mW}$ pump beam was incident at $25^{\circ}$ to the surface normal and focused to a $100-\mu \mathrm{m}$ spot (full width at half maximum perpendicular to the plane of incidence) using reflecting optics. This corresponds to a pump fluence of $\sim 40 \mu \mathrm{J} \mathrm{cm}^{-2}$, two orders of magnitude lower than that used to study nonclassical behavior in Refs. 12 and 13 but similar to that used in Refs. 15-18. An orthogonally polarized, $40 \mathrm{~mW}$ probe beam was incident at $45^{\circ}$ and focused to a $50-\mu \mathrm{m}$ diameter spot centered on that of the pump. The reflected probe beam was recollimated and used to measure the anisotropic modulation of the dielectric function by analyzing the change in its polarization state using a quarter wave plate, polarizing beam splitter cube, and a pair of biased photodiodes.

Although REOS differs somewhat from the polarization insensitive reflectivity technique used by Misochko et al. ${ }^{15-18}$ the phonon contribution to the dielectric function is probed in the same way and we obtain very similar values for the differential reflectivity. The transient difference in the intensity of the orthogonal probe polarizations was measured as a difference in the two photodiode currents, $\Delta I$, as a function of pump-probe delay. The time-averaged photocurrents were maintained in the balance condition required for effective noise canceling using a feedback loop to adjust the gain in one $\mathrm{arm}^{22}$ with a time constant of $100 \mathrm{~s}$. The samples were rotated about the surface normal to achieve the maximum phonon contribution to the signal. The photocurrent in a single arm of the bridge, $I_{\mathrm{o}}$, was approximately $3 \mathrm{~mA}$ which gives a shot-noise detection limit for a fractional change in $I$, $\Delta I_{\mathrm{n}} / I_{\mathrm{o}}=\left(4 e / I_{\mathrm{o}}\right)^{1 / 2}$, of $1.5 \times 10^{-8} / \sqrt{\mathrm{Hz}}$. To closely approach this limit the optical noise, which was dominated by intensity fluctuations in the argon ion pump laser, was reduced by making use of the filtering action of the Ti:sapphire gain medium at megahertz $(\mathrm{MHz})$ frequencies. The pump beam was chopped at $1.5 \mathrm{MHz}$ using a fused silica acousto-optic modulator and the difference in photodiode currents measured using a radio-frequency lock-in amplifier (Stanford Research model SR844). A pair of fused silica prisms compensated for the second-order dispersion introduced by the modulator in the pump beam and an identical length of fused silica in the probe beam. The resulting pump and probe pulse duration at the sample, measured by interferometric autocorrelation, was $12 \mathrm{fs}$.

Time domain REOS signal traces for $\mathrm{Bi}$ and GaAs at room temperature are shown in Fig. 1. In the case of $\mathrm{Bi}$ the
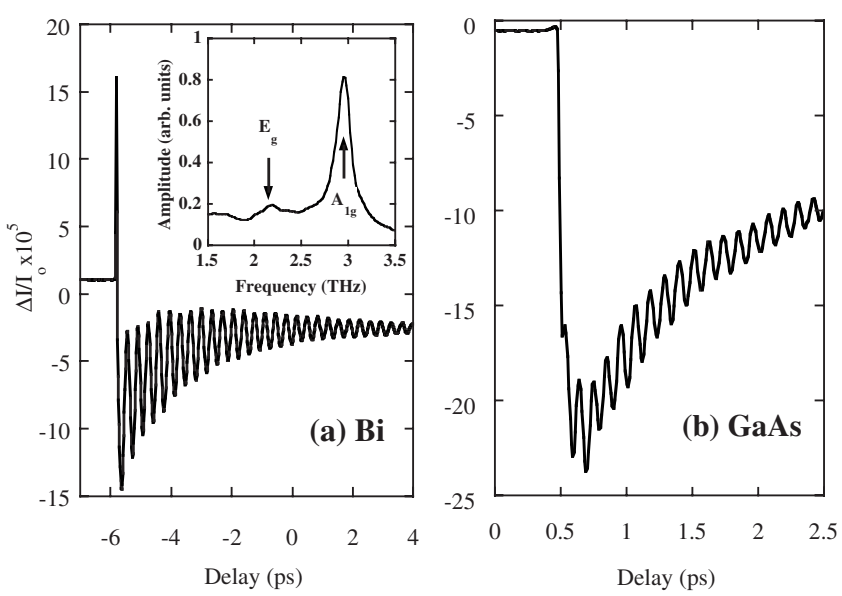

FIG. 1. REOS signals as a function of pump-probe delay for (a) $\mathrm{Bi}$ and (b) GaAs. Inset in (a) shows spectrum of $\Delta I(t)$.

oscillatory part of the signal mainly arises from impulsive excitation of a fully symmetric $\left(A_{1 \mathrm{~g}}\right)$, Raman-active phonon with a frequency near $2.9 \mathrm{THz}$. From symmetry considerations, ${ }^{23}$ the contribution of this mode to the anisotropic reflectivity change should be weak but in fact we find that the differential reflectivity is comparable with previous studies of the isotropic reflectivity for the same excitation fluence and wavelength. ${ }^{15}$ Compared with previous REOS studies of polycrystalline Bi films prepared by evaporation ${ }^{24}$ the magnitude of the differential signal from the $A_{1 \mathrm{~g}}$ mode is similar but that from the $E_{\mathrm{g}}$ mode near $2.2 \mathrm{THz}$ [see inset in Fig. 1(a)] is weaker, presumably because of differences in crystallographic texture. The phonon excitation mechanism is thought to be displacive. ${ }^{1}$ In the case of GaAs, a polar longitudinal optical phonon with a frequency near $8.8 \mathrm{THz}$ is excited by the transient polarization associated with screening currents in the high field intrinsic region. ${ }^{2}$

We now discuss details of the noise measurements. Lock-in amplifiers can be used to measure noise in a signal, either by statistical analysis of a set of repeated measurements or by using the built-in noise measurement function of digital instruments. In the second approach, which is less affected by system drift and therefore preferred, the moving average of the signal is typically computed and subtracted from the current value to obtain the deviation. The mean average deviation is then obtained as a moving average of the absolute value of the deviations, which for Gaussian noise is related to the rms deviation by a constant factor. In the lock-in amplifier used in the present study the moving averages have an exponential time constant that varies between 10 and 80 times that of the lock-in low pass filter which means that a very long settling time is necessary to make accurate noise measurements. In the context of our measurements, the settling time needs to be less than the time between steps in pump-probe delay, which we call the dwell time.

In order to characterize the noise-measurement procedure it is desirable to study a simpler process than REOS so that the results can more easily be interpreted. For this purpose we chose to measure the terahertz electric field produced by difference frequency generation in GaSe using electro-optic 

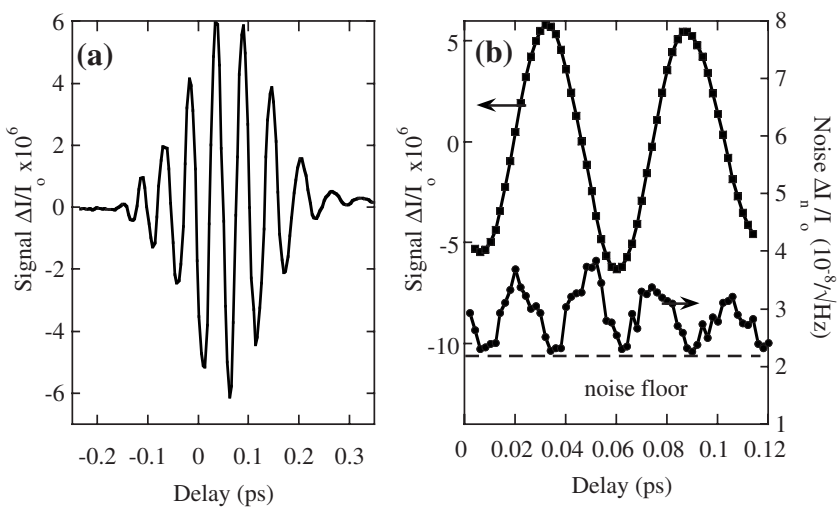

FIG. 2. (a) Terahertz emission from GaSe detected by electrooptic sampling. (b) Signal and noise measurements performed over a two cycle portion of (a). In (b) the noise is measured with a 10-ms lock-in time constant and an interval of $10 \mathrm{~s}$ between consecutive data points. The horizontal dashed line indicates the noise floor in the absence of the pump beam.

sampling in a 40- $\mu \mathrm{m}$ thick [110] ZnTe crystal $^{25}$ and the same polarization analysis system. We do not expect any phasedependent noise in this simple second-order nonlinear process except possibly as a result of fluctuations in pump-probe delay. An example of the signal obtained from a $100-\mu \mathrm{m}$-thick GaSe crystal at a phase matching angle of $42^{\circ}$ is shown in Fig. 2(a). Figure 2(b) displays the fractional signal and noise measured over a smaller range of delay with a time constant of $10 \mathrm{~ms}$ and a $10 \mathrm{~s}$ interval between successive 2 -fs increments in delay. The noise peaks where the signal changes most rapidly and is close to the noise floor in the absence of the pump beam at intermediate delays. This behavior can be explained by the existence of a pump-probe timing jitter of rms magnitude $0.04 \mathrm{fs} / \sqrt{\mathrm{Hz}}$ arising from beam pointing instability and asymmetric optical paths. If the time constant is decreased or the dwell time increased then the peaks in noise at the nodes in the signal are not reduced but if the changes are reversed then they become much larger. This is illustrated in Fig. 3 which shows the average noise in a delay line scan averaged over approximately two cycles as a function of time constant for a dwell time of $1 \mathrm{~s}$. The average noise decreases with decreasing time constant and levels off when the time constant is one thousand times smaller than the dwell time. This ratio was therefore chosen for the REOS noise measurements.

Figures 4(a) and 4(b) show the results of REOS measurements on $\mathrm{Bi}$ and $\mathrm{GaAs}$, respectively. The signal and noise are displayed over a smaller interval of delay than shown in Fig. 2 and the data sets are different. The noise was measured at each step in delay using the built-in noise function of the lock-in amplifier with a time constant of $3 \mathrm{~ms}$, a dwell time of $3 \mathrm{~s}$ and a $24 \mathrm{~dB} /$ octave low pass filter roll off, following the protocol for accurate measurement established above. The noise power was subsequently averaged over six successive delay scans. The main finding is that the measurements do not show any phase-dependent noise signature associated with the optically excited phonons. In both cases the noise is higher than that measured in the absence of the pump [dashed lines in Figs. 4(a) and 4(b)] because of the effects of scattering of a small amount of the pump into the probe

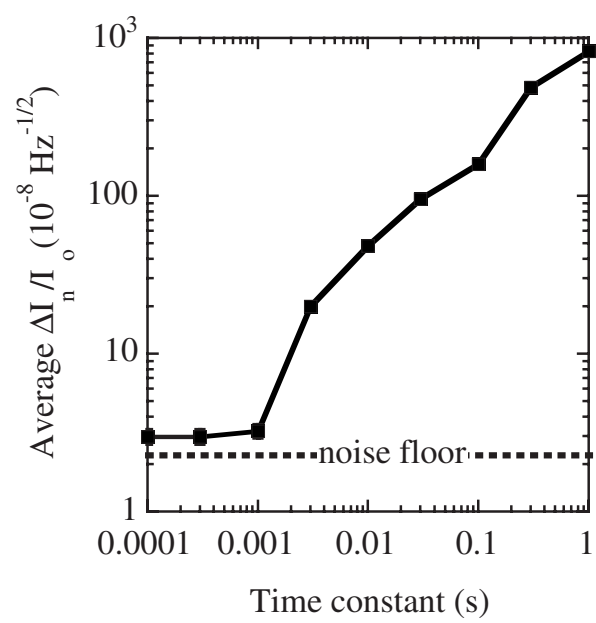

FIG. 3. Fractional noise in electro-optic sampling measurements of terahertz emission from GaSe as a function of lock-in amplifier time constant with an interval of $1 \mathrm{~s}$ between points. The noise signal is averaged over two cycles of the terahertz signal. The horizontal dashed line indicates the noise floor in the absence of the pump beam.

beam at surface imperfections and pump-probe timing jitter. Estimates of the latter are shown by the dotted curves in Fig. 4. In the absence of the pump beam, the noise in $\Delta I / I_{0}$, $\Delta I_{\mathrm{n}} / I_{\mathrm{o}}$, was $2.5 \times 10^{-8} / \sqrt{\mathrm{Hz}}$ for the GaAs sample and 3.2 $\times 10^{-8} / \sqrt{\mathrm{Hz}}$ for Bi. The fractional dark noise due to the electronics alone was $1.4 \times 10^{-8} / \sqrt{\mathrm{Hz}}$ so that the noise in the GaAs case is close to that expected from combining the shot noise of $1.5 \times 10^{-8} / \sqrt{\mathrm{Hz}}$ with the electronic noise. The higher value for $\mathrm{Bi}$ is due to a less perfect sample surface. If the lock-in time constant is significantly increased or if the dwell timed is reduced then peaks in the noise emerged at nodes in the signal as a result of too small a settling time, suggesting that the results in Refs. 15-18 are a measurement artifact. The increase in the ratio of the standard deviation to the peak amplitude with phonon frequency evident in these reports is easily explained in this picture. In the previous measurements, ${ }^{15}$ the maximum standard deviation in the phonon contribution to the signal was approximately $50 \%$ of
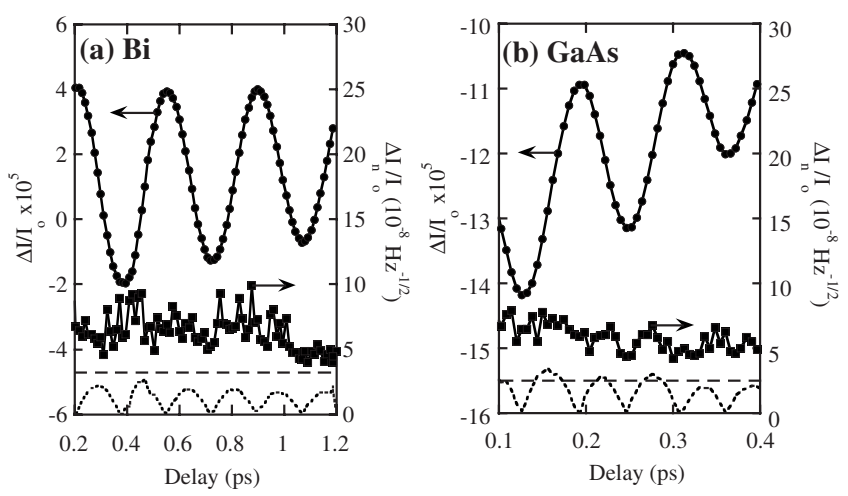

FIG. 4. Fractional REOS signal, $\Delta I / I_{0}$, and fractional noise in $I$, $\Delta I_{\mathrm{n}} / I_{\mathrm{o}}$, for (a) Bi and (b) GaAs. The dotted curve at the bottom of each panel shows the estimated contribution to the noise from timing jitter and the horizontal dashed lines indicate the noise floor in the absence of the pump beam. 
the peak to peak phonon contribution to the probe signal for $\mathrm{Bi}$ and $20 \%$ for GaAs. If we assume a lock-in amplifier time constant of $1 \mathrm{~s}$ and a $24 \mathrm{~dB} /$ octave low pass filter so as to calculate the rms noise in a typical experiment using a motorized delay line then we can place an upper limit on any phase-dependent contribution to the ratio of the noise to the signal of $0.04 \%$ for $\mathrm{Bi}$ and $0.06 \%$ for GaAs. Thus we find that any phase-dependent noise must be at least 40-50 dB smaller than previously suggested in Ref. 15.

It is also possible to make repeated measurements of the signal at a fixed delay in order to compute the standard deviation, as done in Refs. 15-18. We performed a few measurements to check for consistency between the two approaches. In this second approach the minimum settling time between consecutive measurements can be evaluated by treating the lock-in as a multiplier followed by an $m$ th-order low pass filter. The variance of the detected noise is

$$
I_{n}^{2}(t)=\int_{-\infty}^{\infty} \alpha(\Delta \omega) \cos ^{2}(\Delta \omega t) G(\Delta \omega) d(\Delta \omega),
$$

where $\alpha$ is proportional to the noise power spectral density, $\Delta \omega$ is the frequency deviation from the modulation frequency, and $G$ is the power transfer function of the filter. For white noise the time varying part of Eq. (1) becomes

$$
I_{n}^{2}(t)=\alpha \int_{-\infty}^{\infty} \frac{\cos (2 \Delta \omega t)}{\left[1+(\Delta \omega \tau)^{2}\right]^{m}} d(\Delta \omega \tau),
$$

where $\tau$ is the lock-in time constant. In a typical delay scan with 10 fs steps, $24 \mathrm{~dB} /$ octave roll off $(m=4)$ and a 300-ms time constant, the fractional signal change between steps is $\sim 5 \times 10^{-6}$ and the typical fractional $\mathrm{rms}$ noise floor is $\sim 1.3 \times 10^{-8}$. Under these conditions, numerical evaluation of Eq. (2) shows that it is necessary to wait more than $10 \tau$ for the rms noise to settle to the noise floor. The settling time is considerably lengthened by reducing the filter order. We tested that this approach to measuring noise was consistent with our previous one by recording the signal amplitude from the Bi sample four hundred times at both nodes and antinodes in the phonon contribution under the conditions stated above. The standard deviation was then calculated and compared with the value obtained using the built-in noise function. We found no significant difference between the two approaches. We note that any long term drift in pump-probe delay would manifest itself as a larger standard deviation at nodes in the signal in this approach even with an appropriate settling time.

In summary, we find no evidence of phase-dependent noise in time-domain reflectivity studies of phonons in $\mathrm{Bi}$ and GaAs excited by nanojoule optical pulses and thus no suggestion that the phonons are anything but coherent. Noise maxima coinciding with nodes in the signal can however be observed as an experimental artifact due to system drift or when using too short an instrument settling time.

We acknowledge the financial support of the Engineering and Physical Science Research Council (EPSRC) and would like to thank Phil Hobbs for advice on optimizing the noise canceling circuit.
*Present address: King Abdullah University of Science and Technology, Physical Sciences and Engineering Division, P.O. Box 55455, Jeddah 21535, Saudi Arabia.

${ }^{\dagger}$ Author to whom correspondence should be addressed; s.r.andrews@bath.ac.uk

${ }^{1}$ T. K. Cheng S. D. Brorson, A. Z. Kazeroonian, J. S. W. Moodera, J. G. Dresselhaus, G. M. S. Dresselhaus, and I. P. Ippen, Appl. Phys. Lett. 57, 1004 (1990).

${ }^{2}$ G. C. Cho, W. Kutt, and H. Kurz, Phys. Rev. Lett. 65, 764 (1990).

${ }^{3}$ R. Merlin, Solid State Commun. 102, 207 (1997).

${ }^{4}$ D. F. Walls, Nature (London) 306, 141 (1983).

${ }^{5}$ R. E. Slusher, L. W. Hollberg, B. Yurke, J. C. Mertz, and J. F. Valley, Phys. Rev. Lett. 55, 2409 (1985).

${ }^{6}$ R. Chiao and J. Garrison, Quantum Optics (OUP, New York, 2008).

${ }^{7}$ X. Hu and F. Nori, Phys. Rev. Lett. 79, 4605 (1997).

${ }^{8}$ X. Hu and F. Nori, Physica B 263-264, 16 (1999).

${ }^{9}$ G. A. Garrett, A. G. Rojo, A. K. Sood, J. F. Whitaker, and R. Merlin, Science 275, 1638 (1997).

${ }^{10}$ G. A. Garrett, J. F. Whitaker, A. K. Sood, and R. Merlin, Opt. Express 1, 385 (1997).

${ }^{11}$ A. Bartels, T. Dekorsy, and H. Kurz, Phys. Rev. Lett. 84, 2981 (2000).

${ }^{12}$ S. L. Johnson, P. Beaud, E. Vorobeva, C. J. Milne, E. D. Murray, S. Fahy, and G. Ingold, Phys. Rev. Lett. 102, 175503 (2009).
${ }^{13}$ O. V. Misochko, M. Hase, K. Ishioka, and M. Kitajima, Phys. Rev. Lett. 92, 197401 (2004).

${ }^{14}$ J. Zhao, A. V. Bragas, D. J. Lockwood, and R. Merlin, Phys. Rev. Lett. 93, 107203 (2004).

${ }^{15}$ O. V. Misochko, K. Sakai, and S. Nakashima, Phys. Rev. B 61, 11225 (2000).

${ }^{16}$ O. V. Misochko, K. Kisoda, K. Sakai, and S. Nakashima, Appl. Phys. Lett. 76, 961 (2000).

${ }^{17}$ O. V. Misochko, K. Kisoda, K. Sakai, and S. Nakashima, Phys. Rev. B 61, 4305 (2000).

${ }^{18}$ O. V. Misochko, Phys. Lett. A 269, 97 (2000).

${ }^{19}$ In a private communication, O. V. Misochko has confirmed that in Refs. 15-18 the variance is plotted using the same units as the square of the amplitude.

${ }^{20}$ T. Dekorsy, T. Pfeifer, W. Kütt, and H. Kurz, Phys. Rev. B 47, 3842 (1993).

${ }^{21}$ S. R. Andrews, A. Armitage, P. G. Huggard, C. J. Shaw, G. P. Moore, and R. Grey, Phys. Rev. B 66, 085307 (2002).

${ }^{22}$ P. C. D. Hobbs, Appl. Opt. 36, 903 (1997).

${ }^{23}$ T. Dekorsy, H. Auer, C. Waschke, H. J. Bakker, H. G. Roskos, H. Kurz, V. Wagner, and P. Grosse, Phys. Rev. Lett. 74, 738 (1995).

${ }^{24}$ M. Hase, K. Mizoguchi, H. Harima, and S. Nakashima, Appl. Phys. Lett. 69, 2474 (1996).

${ }^{25}$ A. Hussain and S. R. Andrews, Appl. Phys. Lett. 88, 143514 (2006). 